\title{
Evaluation of ivc and ijv dimensions in prediction of fluid responsiveness in spontaneous breathing patients with septic shock
}

\author{
A Hasanin, A Lotfy", IR Abdel-Aal, J Elkholey, A El-Adawy, H Mohamed, A Zaghlol, M Salem, A Mukhtar \\ From ESICM LIVES 2015 \\ Berlin, Germany. 3-7 October 2015
}

\section{Introduction}

Prediction of fluid responsiveness (FR) is a critical step in management of patients with septic shock. Using ultrasound in detection of inferior vena cava (IVC) diameters and collapsibility is established in mechanically ventilated patients; however its use in spontaneous breathing patients is still controversial [1]. Few studies reported a correlation between internal jugular vein dimensions and central venous pressure (CVP) $[2,3]$ but no data are available about the use of IJV dimensions in detection of FR.

\section{Objectives}

The aim of our study is to determine the possible rule of IVC diameters, collapsibility, and IJV dimensions in prediction of FR in spontaneous breathing patients.

\section{Methods}

Twenty spontaneous breathing patients with septic shock were included in the study. Ultrasound examination was done before fluid resuscitation. IVC minimal and maximum diameters, IVC collapsibility index (IVC maximum IVC minimum/IVC maximum), IJV cross sectional area, IJV/Common carotid artery (CCA) ratio, and IJV aspect ratio (IJV vertical diameter/IJV transverse diameter) were measured before fluid resuscitation. Transthoracic echocardiography (TTE) was done to determine FR which was defined as increase in sub-aortic velocity time integral (VTI) $>15 \%$ after fluid bolus $7 \mathrm{ml} / \mathrm{Kg}$. Sensitivity, Specificity and Area under receiver operating characteristic (AUROC) curves were determined for all ultrasound parameters as well as CVP for detection of FR.

\section{Results}

Eleven patients showed FR and nine patients were nonresponders. APACHE II score was $16 \pm 6.3$, age was $36 \pm 15$ years. AUROC curve $(95 \% \mathrm{CI})$ for prediction of FR was: 0.51 (0.27-0.73) for CVP with sensitivity $27 \%$ and specificity $100 \%$ at cutoff value $9 \mathrm{mmHg}, 0.5(0.27-0.72)$ for IJV aspect ratio, $0.56(0.33-0.78)$ for IJV/CCA, 0.54(0.31-0.77) for IJV surface area, $0.52(0.28-0.74)$ for IVC collapsibility index, 0.72(0.53-0.92) for IVC minimum diameter with sensitivity $45 \%$ and specificity $89 \%$ at cutoff value $0.3 \mathrm{~cm}$, $0.76(0.48-0.90)$ for IVC maximum diameter with sensitivity $82 \%$ and specificity $67 \%$ at cutoff value $1.5 \mathrm{~cm}$.

\section{Conclusions}

Our finding supports the evidence of the poor value of most of static parameters in prediction of FR in spontaneous breathing patients. IVC minimum and maximum diameters were the best parameters in detection of FR in our cohort.

\section{Published: 1 October 2015}

\section{References}

1. Muller L, Bobbia X, Toumi M, Louart G, Molinari N, Ragonnet B, et al: Respiratory variations of inferior vena cava diameter to predict fluid responsiveness in spontaneously breathing patients with acute circulatory failure: need for a cautious use. Crit Care 2012, 16(5):R188.

2. Bailey JK, McCall J, Smith S, Kagan RJ: Correlation of internal jugular vein/ common carotid artery ratio to central venous pressure: a pilot study in pediatric burn patients. J Burn Care Res 2012, 33(1):89-92.

3. Keller AS, Melamed R, Malinchoc M, John R, Tierney DM, Gajic O: Diagnostic accuracy of a simple ultrasound measurement to estimate central venous pressure in spontaneously breathing critically ill patient. J Hosp Med 2009, 4(6):350-355.

doi:10.1186/2197-425X-3-S1-A594

Cite this article as: Hasanin et al:: Evaluation of ivc and ijv dimensions in prediction of fluid responsiveness in spontaneous breathing patients with septic shock. Intensive Care Medicine Experimental 2015 3(Suppl 1):A594. 